PHYSICAL REVIEW D 82, 024009 (2010)

\title{
New class of accelerating black hole solutions
}

\author{
Joan Camps ${ }^{1, *}$ and Roberto Emparan ${ }^{1,2, \dagger}$ \\ ${ }^{1}$ Departament de Física Fonamental and Institut de Ciències del Cosmos, Universitat de Barcelona, \\ Martí i Franquès 1, E-08028 Barcelona, Spain \\ ${ }^{2}$ Institució Catalana de Recerca i Estudis Avançats (ICREA), Passeig Lluís Companys 23, E-08010 Barcelona, Spain
}

(Received 17 May 2010; published 13 July 2010)

\begin{abstract}
We construct several new families of vacuum solutions that describe black holes in uniformly accelerated motion. They generalize the $C$ metric to the case where the energy density and tension of the strings that pull (or push) on the black holes are independent parameters. These strings create large curvatures near their axis and when they have infinite length they modify the asymptotic properties of the spacetime, but we discuss how these features can be dealt with physically, in particular, in terms of "wiggly cosmic strings." We comment on possible extensions and extract lessons for the problem of finding higher-dimensional accelerating black hole solutions.
\end{abstract}

DOI: 10.1103/PhysRevD.82.024009

\section{INTRODUCTION}

The $C$ metric is a solution of the Einstein equations that describes the spacetime of two black holes uniformly accelerating in opposite directions [1]. This solution and its variants have been applied to a number of interesting problems, including gravitational radiation from accelerated sources [2], instantonic pair creation of black holes [3], black holes on branes [4], five-dimensional black rings [5], etc. It is remarkable that a simple, exact solution is available for the study of such a variety of problems, and so it seems desirable to investigate possible extensions of it.

The generalization that we study in this paper can be easily motivated. In the original $C$ metric, conical singularities are unavoidable since they reflect the need of an external force to accelerate the black holes. Conical deficit angles correspond to distributional stringlike sources with linear energy density $\varepsilon$ and tension $T=\varepsilon$. The black holes in the $C$ metric are then accelerated under the pull of two such semi-infinite strings. These sources are physically appealing since they can be made sense of as cosmic strings (vortices) in the limit in which their thickness is negligible. Note, however, that for the purpose of pulling on the black holes one merely needs a tensile string. In particular, it is not necessary that its energy density equals its tension. Thus it appears that a one-parameter generalization of the $C$ metric where the energy density of the string is independent of its tension should be possible. Our purpose is to describe a solution where black holes are accelerated by such generic strings.

Stringlike sources with $\varepsilon \neq T$ arise as the zero-thickness limit of a variety of less singular sources, such as "wiggly" cosmic strings or cylindrical shells. A main difference with the $\varepsilon=T$ sources is that when $\varepsilon \neq T$ the Newtonian gravitational potential does not vanish and the gravitational

\footnotetext{
*jcamps@ub.edu

†emparan@ub.edu
}

PACS numbers: 04.70.Bw, 04.20.Jb, 04.50.Gh, 11.27.+d

field has nontrivial local curvature. As a consequence the spacetime is not asymptotically flat, not even locally. Although this may appear as a serious drawback, it need not be a problem in physical situations in which the string is not infinite but forms a (possibly long) loop, whose radius provides a natural cutoff for the geometry at large transverse distance from the string (this is often assumed also for e.g., global strings). One might also take the view that, like e.g., for the Melvin universe, these strings define their own asymptotic class. We shall accept that these solutions admit physical motivation.

Interestingly, we can also have "struts" that stretch between the black holes and push them apart. These solutions are locally inequivalent to those with pulling strings and since the struts have finite length, the asymptotic behavior at spatial infinity is expected to be better. The struts have negative tension, but in contrast to the $C$ metric, they do not violate any energy condition if the energy density on the struts is large enough. There is also the possibility of solutions with both strings and struts. Although the physical import of these metrics is less clear, they provide the largest (five-parameter) family of solutions in this class and we present them explicitly for completeness.

There are several interesting possible extensions of the solutions described in this paper. In fact one of the reasons leading us to their study has been the consideration of higher-dimensional generalizations of the $C$ metric. In the context of these elusive solutions, which have been sought for many of the applications mentioned in our opening paragraph, the possibility of different kinds of string sources is potentially even more important than in four dimensions. We shall address this point toward the end of the paper.

In the next section we analyze the basic string solutions that later will be used to pull, or push, on the black holes. In Sec. III we present the new metrics for accelerating black holes, first with pulling strings, then with pushing struts, 
and we analyze their main properties. We also present the most general solution with both strings and struts. Section IV discusses extensions of these solutions. The Appendix contains an alternative form for the new metrics that resembles more closely the conventional way of writing the $C$ metric.

\section{THE LEVI-CIVITA STRING AND ITS SOURCES}

The Levi-Civita spacetime ${ }^{1}$

$$
d s^{2}=-\rho^{2 m} d t^{2}+\rho^{2 m(m-1)}\left(d z^{2}+d \rho^{2}\right)+\rho^{2(1-m)} \frac{d \phi^{2}}{C^{2}},
$$

is a long-known cylindrically symmetric solution to the vacuum Einstein equations [6]. It is a Weyl metric of general Petrov type, ${ }^{2}$ and contains two parameters: $m$, which determines the local curvature, and $C$, which introduces a conical structure along the axis when the normalization of $\phi$ is fixed by identifying $\phi \sim \phi+2 \pi$. When $m=0,1$ with $C=1$ it reproduces Minkowski and Rindler space, respectively, but for generic values of $m$ and $C$ the solution is not asymptotically flat as $\rho \rightarrow \infty$ and exhibits a curvature singularity at $\rho=0$. As a Weyl solution, it corresponds to an infinite line source of the Newtonian potential with linear density $m / 2 G$. We shall refer to it as the "Levi-Civita string".

It has been argued that, in order to admit an interpretation as the spacetime of a cylindrical source, one must have $m<1 / 2$, or possibly $m<1$ (see e.g., [7,8] and references therein). The precise range will not concern us much, since later we shall be mostly interested in small values of $m$, as well as $C$ close to 1 , for which the line source is readily interpreted. Nevertheless let us briefly discuss the general case. The singular behavior near the axis $\rho=0$ may be smoothed by replacing the region around it with an extended source, and a simple example is a cylindrical tubular shell [9-14]. Cutting the metric at $\rho=\rho_{s}$ and replacing the interior with flat Minkowski spacetime $(m=$ $0, C=1$ ), one can apply Israel's analysis [15] to obtain the stress tensor at the shell interface

$$
\begin{aligned}
T_{t}^{t} & =\frac{\rho_{s}^{m-1}}{8 \pi G}\left((1-m)^{2} \rho_{s}^{-m^{2}}-C\right), \\
T_{z}^{z} & =\frac{\rho_{s}^{m-1}}{8 \pi G}\left(\rho_{s}^{-m^{2}}-C\right), \\
T_{\phi}^{\phi} & =\frac{\rho_{s}^{m-1}}{8 \pi G} m^{2} \rho_{s}^{-m^{2}} .
\end{aligned}
$$

In this manner, the problem of interpreting the strong curvature singularity at $\rho=0$ is shifted to that of finding

\footnotetext{
${ }^{1}$ The solution does not contain any length scale so the coordinates may be regarded as normalized relative to an arbitrary length unit.

${ }^{2}$ Except for $m=0,1 / 2, \pm 1,2$.
}

an adequate source that smoothens the milder singularity at the shell. Observe in (2.2) the presence of not only energy density and tension along $z$, but also a hoop stress $T_{\phi}^{\phi}$, which seems to be a necessary feature of any possible source of these spacetimes. Typically the equation of state of the shell matter will impose a relationship between $m, C$, and $\rho_{s}$. We shall not dwell much on candidate shell sources, but merely note that tubular structures with similar properties appear naturally in string theory in the form of supertubes and closely related helical strings (smeared along the $z$ direction). It seems likely that combinations or variants of these can provide adequate sources for these spacetimes.

From (2.2) we can introduce the energy density per unit length [13],

$$
\varepsilon=-\int_{\rho=\rho_{s}} d \phi \sqrt{g_{\phi \phi}} T_{t}^{t}=\frac{1}{4 G}\left(1-\frac{(1-m)^{2}}{C \rho_{s}^{m^{2}}}\right)
$$

and tension

$$
T=-\int_{\rho=\rho_{s}} d \phi \sqrt{g_{\phi \phi}} T_{z}^{z}=\frac{1}{4 G}\left(1-\frac{1}{C \rho_{s}^{m^{2}}}\right) .
$$

These are nontrivially equal only in the case of a conicaldefect spacetime, $m=0, C \neq 1$.

Let us now consider the Levi-Civita spacetime (2.1) expanded to linear order in $m$ and in $\gamma \equiv C-1$,

$$
\begin{aligned}
d s^{2} \simeq & -(1+2 m \log \rho) d t^{2}+(1-2 m \log \rho)\left(d z^{2}+d \rho^{2}\right) \\
& +(1-2 m \log \rho)(1-2 \gamma) \rho^{2} d \phi^{2} .
\end{aligned}
$$

In this linearized approximation we can also write $m$ and $\gamma$ in terms of the energy density (2.3) and tension (2.4) as

$$
m \simeq 2 G(\varepsilon-T), \quad \gamma \simeq 4 G T .
$$

We will regard these simple relations as the basic interpretation of the parameters of the Levi-Civita string. Observe that $\rho_{s}$ does not appear in them. Relatedly, the hoop stress $T_{\phi}^{\phi}$ is $O\left(\mathrm{~m}^{2}\right)$ and therefore it does not appear in the linearized approximation.

Using (2.6), and performing the coordinate change

$$
\begin{aligned}
(1-4 G(\varepsilon+T) \log r) r^{2}= & (1-8 G T)(1-4 G(\varepsilon-T) \\
& \times \log \rho) \rho^{2}
\end{aligned}
$$

(to the required expansion order) the metric (2.5) is brought to the form

$$
\begin{aligned}
d s^{2} \simeq & -(1+4 G(\varepsilon-T) \log r) d t^{2}+(1-4 G(\varepsilon-T) \\
& \times \log r) d z^{2}+(1-4 G(\varepsilon+T) \log r)\left(d r^{2}+r^{2} d \phi^{2}\right) .
\end{aligned}
$$

This can be recognized as the solution to the linearized Einstein equations, in transverse gauge, 


$$
\square\left(h_{\mu \nu}-\frac{h}{2} \eta_{\mu \nu}\right)=-16 \pi G T_{\mu \nu}
$$

with distributional stress tensor

$$
T_{\mu \nu}=\operatorname{diag}(\varepsilon,-T, 0,0) \delta(x) \delta(y)
$$

which confirms our interpretation of $\varepsilon$ and $T$.

The solution (2.8) has been previously studied in the context of a different kind of string source, namely, "wiggly strings" (see e.g., [16]). Cosmic strings have Lorentzinvariant world sheets so $\varepsilon=T$, but if they acquire a shortdistance structure (wiggles), then when this is averaged it produces an effective linear source with $\varepsilon \neq T$. This is an appealing physical realization of this linearized spacetime.

Finally, observe that the tension of the string may be negative, and hence the string exerts pressure, while satisfying the usual energy conditions if $\varepsilon$ is large enough. We will refer to this as the "Levi-Civita strut." Let us discuss it in the case of small $m$ and small $C-1$. Equation (2.6) implies that, when $C<1$ and hence $\gamma<0$,

$$
\varepsilon \simeq \frac{m-|\gamma| / 2}{2 G}, \quad \varepsilon-T \simeq \frac{m}{2 G}, \quad \varepsilon-|T| \simeq \frac{m-|\gamma|}{2 G} .
$$

Therefore the weak, strong, and dominant energy conditions are all satisfied if $m>|\gamma|$. While it does not seem possible to realize these struts in terms of wiggly cosmic strings, one may still obtain them from tubular shells. They might be elastically unstable due to the negative tension, but presumably this depends on the specific shell matter (see [13] for energy conditions on generic shells).

\section{ACCELERATING BLACK HOLES}

We describe different families of solutions where the black holes are accelerated either by strings that pull or by struts that push on them. They all contain the $C$ metric as a limit.

\section{A. Pulling with strings}

We construct the metric using conventional integrability techniques for Weyl spacetimes (see e.g., [7,17]). The rod structure for the solution ${ }^{3}$ is depicted in Fig. 1. The metric reads

$$
d s^{2}=-e^{2 U} d t^{2}+e^{2 \nu}\left(d z^{2}+d \rho^{2}\right)+e^{-2 U} \rho^{2} \frac{d \phi^{2}}{C^{2}}
$$

with

$$
e^{2 U}=\rho^{2 m} \frac{\mu_{1}^{1-m} \mu_{3}}{\mu_{2}},
$$

and

\footnotetext{
${ }^{3}$ That is, the line sources for the Newtonian potential $U$.
}

$$
a_{1} \quad a_{2} \quad a_{3}
$$

FIG. 1. Weyl rod structure for the solution with an accelerating black hole pulled by a semi-infinite Levi-Civita string. The rod at $z<a_{1}$ has linear density $m / 2 G$. The rods at $a_{1}<z<a_{2}$ and $z>a_{3}$ have linear density $1 / 2 G$.

$$
\begin{aligned}
e^{2 v}= & \rho^{2 m(m-1)}\left(\frac{\mu_{1}}{\left(\mu_{1}^{2}+\rho^{2}\right)^{1-m}}\left(\frac{\mu_{1} \mu_{2}+\rho^{2}}{\mu_{1} \mu_{3}+\rho^{2}}\right)^{2}\right)^{1-m} \\
& \times \frac{\mu_{3}\left(\mu_{2} \mu_{3}+\rho^{2}\right)^{2}}{\mu_{2}\left(\mu_{2}^{2}+\rho^{2}\right)\left(\mu_{3}^{2}+\rho^{2}\right)},
\end{aligned}
$$

where

$$
\mu_{i}=a_{i}-z+\sqrt{\left(a_{i}-z\right)^{2}+\rho^{2}} .
$$

The solution contains five parameters: $m, C, a_{i}(i=1,2$, 3 ). One of the $a_{i}$ may be absorbed by a shift in $z$ so only their differences $a_{i}-a_{j}$ are physical. The parameter $C$ will be fixed presently by a regularity condition, so in the end we will be left with a three-parameter family of solutions. It contains the $C$ metric as the particular case $m=0$ (see the Appendix), while for $m=+1$ we obtain a double Wick rotation of the Schwarzschild spacetime. When all the $a_{i} \rightarrow+\infty$ the solution reduces, after a rescaling of coordinates, to the Levi-Civita spacetime. Unlike the $C$ metric, for generic values of the parameters the solution is not algebraically special.

The rod structure allows a ready interpretation of the solution. Along the axis $\rho=0$, we expect to have the following:

(i) A semi-infinite Levi-Civita string at $z<a_{1}$ with rod density $m / 2 G$.

(ii) A black hole horizon at $a_{1}<z<a_{2}$.

(iii) An "exposed" axis of rotation at $a_{2}<z<a_{3}$.

(iv) An acceleration (Rindler) horizon at $a_{3}<z$.

We proceed to analyze the solution near each of these rods.

Exposed axis.-Assuming that $\phi \sim \phi+2 \pi$, the absence of conical singularities at the exposed axis at $\{\rho=$ $0, a_{2}<z<a_{3}$ \} requires that

$$
C=\left.\lim _{\rho \rightarrow 0} e^{-(U+\nu)}\right|_{a_{2}<z<a_{3}}=\frac{1}{2^{m}} \frac{\left(a_{3}-a_{1}\right)^{1-m}}{a_{3}-a_{2}} .
$$

Black hole horizon.-Near the rod at $a_{1}<z<a_{2}$, to leading order in $\rho$ the metric is

$$
\begin{aligned}
d s^{2} \simeq & \left(2\left(z-a_{1}\right)\right)^{m-1} \frac{a_{3}-z}{a_{2}-z}\left(-\rho^{2} d t^{2}+\left(\frac{a_{2}-a_{1}}{a_{3}-a_{1}}\right)^{2(1-m)}\right. \\
& \left.\times\left(d z^{2}+d \rho^{2}\right)\right)+\frac{a_{2}-z}{a_{3}-z} \frac{d \phi^{2} / C^{2}}{\left(2\left(z-a_{1}\right)\right)^{m-1}}
\end{aligned}
$$

The horizon at $\rho=0$ is a regular surface away from the Levi-Civita singularity at its pole $z=a_{1}$. The horizon area is 


$$
\begin{aligned}
A_{\mathrm{BH}} & =\left.\int_{0}^{2 \pi} d \phi \int_{a_{1}}^{a_{2}} d z \sqrt{g_{z z} g_{\phi \phi}}\right|_{\rho=0} \\
& =\frac{2 \pi}{C}\left(a_{2}-a_{1}\right)\left(\frac{a_{2}-a_{1}}{a_{3}-a_{1}}\right)^{1-m} \\
& =2^{1+m} \pi\left(a_{3}-a_{2}\right) \frac{\left(a_{2}-a_{1}\right)^{2-m}}{\left(a_{3}-a_{1}\right)^{2-2 m}} .
\end{aligned}
$$

We can compute the surface gravity at the horizon of the Killing vector $\partial_{t}$,

$$
\kappa_{\mathrm{BH}}=\left.\lim _{\rho \rightarrow 0} \frac{\partial_{\rho} \sqrt{-g_{t t}}}{\sqrt{g_{\rho \rho}}}\right|_{a_{1}<z<a_{2}}=\left(\frac{a_{3}-a_{1}}{a_{2}-a_{1}}\right)^{1-m} .
$$

One should keep in mind that given the unusual asymptotics created by the Levi-Civita string, the normalization of the Killing generator of the horizon is somewhat arbitrary. Observe that the product

$$
\kappa_{\mathrm{BH}} A_{\mathrm{BH}}=\frac{2 \pi}{C}\left(a_{2}-a_{1}\right)
$$

is equal to the length of the black hole rod times a factor that accounts for the modified length of the orbits of $\phi$. One might want to regard this as a Smarr-type relation $\kappa_{\mathrm{BH}} A_{\mathrm{BH}}=4 \pi G M$ that would define the mass of the black hole. Actually, this definition of mass is equivalent to the Komar mass of the black hole on its horizon. However, it is unclear to what extent this definition of black hole mass is appropriate in the present context. ${ }^{4}$

Acceleration horizon.-Near $\rho=0$ and $a_{3}<z$ we find

$$
\begin{aligned}
d s^{2} \simeq & \left(2\left(z-a_{1}\right)\right)^{m-1} \frac{z-a_{2}}{z-a_{3}}\left(-\rho^{2} d t^{2}+d z^{2}+d \rho^{2}\right) \\
& +\frac{z-a_{3}}{z-a_{2}} \frac{d \phi^{2} / C^{2}}{\left(2\left(z-a_{1}\right)\right)^{m-1}} .
\end{aligned}
$$

There is an infinite Killing horizon (Rindler) at $\rho=0$ generated by $\partial_{t}$. The apparent singularity at $z=a_{3}$ is just a coordinate artifact and the horizon is regular everywhere. We compute the surface gravity as was done for the black hole horizon,

$$
\kappa_{R}=1
$$

The acceleration of the black hole is ambiguous in that it depends on the normalization of $\partial_{t}$, which for a spacetime with a Levi-Civita string is unclear, and also because the black hole is an extended object. In the case of the $C$ metric, when the black hole is small $\left(a_{2}-a_{1} \ll a_{3}-\right.$ $\left.a_{2}\right)$ its acceleration relative to static asymptotic observers can be unambiguously identified to leading order as $A \simeq$ $\left(2 a_{3}-\left(a_{1}+a_{2}\right)\right)^{-1}$.

The ambiguities in the normalization of $\kappa$ cancel when we consider the quotient

\footnotetext{
${ }^{4}$ See [18] for a definition of "boost mass."
}

$$
\frac{\kappa_{R}}{\kappa_{\mathrm{BH}}}=\left(\frac{a_{2}-a_{1}}{a_{3}-a_{1}}\right)^{1-m} \text {. }
$$

The surface gravities can be associated as usual to horizon temperatures, $T_{\mathrm{BH}, R}=\kappa_{\mathrm{BH}, R} / 2 \pi$. Since $T_{R}<T_{\mathrm{BH}}$ the two temperatures are never equal. Thus, even if the Levi-Civita singularities at the string and infinity could be disposed of, an otherwise regular Euclidean instanton could not be constructed.

Levi-Civita string.-For small $\rho$ and $z<a_{1}$ we have

$$
\begin{aligned}
e^{2 U} & \simeq \rho^{2 m} 2^{1-m} \frac{\left(a_{1}-z\right)^{1-m}\left(a_{3}-z\right)}{a_{2}-z} \\
e^{2 \nu} & \simeq \rho^{2 m(m-1)} \frac{a_{3}-z}{a_{2}-z}\left(2^{2 m-1} \frac{\left(a_{1}-z\right)^{2 m-1}\left(a_{2}-z\right)^{2}}{\left(a_{3}-z\right)^{2}}\right)^{1-m} .
\end{aligned}
$$

The radial dependence is like in (2.1), but there is a dependence on $z$ as well. However, away from the string end point at $z=a_{1}$ these functions vary slowly with $z$. Thus let us introduce, at any given $z$ along the string, the functions $\hat{U}(z)$ and $\hat{\nu}(z)$ by

$$
\begin{aligned}
e^{2 \hat{U}(z)} & =2^{1-m} \frac{\left(a_{1}-z\right)^{1-m}\left(a_{3}-z\right)}{a_{2}-z}, \\
e^{2 \hat{\nu}(z)} & =\frac{a_{3}-z}{a_{2}-z}\left(2^{2 m-1} \frac{\left(a_{1}-z\right)^{2 m-1}\left(a_{2}-z\right)^{2}}{\left(a_{3}-z\right)^{2}}\right)^{1-m} .
\end{aligned}
$$

These are approximately constant in a neighborhood of a given $z$ not close to $a_{1}$, so we may locally absorb them through a change of coordinates in such a way that the geometry is well approximated by a metric of the form (2.1) with a $z$-dependent $C$ parameter ${ }^{5}$

$$
\hat{C}(z)=C e^{\hat{U}(z)} e^{\hat{\nu}(z)\left[(1-m) /\left(m^{2}-m+1\right)\right]} .
$$

This is a monotonically decreasing function of $z$. In this sense, we may say that the string tension increases along the string from infinity toward the black hole. Note that for the $C$ metric with $m=0$ this $z$ dependence cancels out. For small $m$

$$
\hat{C}(z)=\frac{a_{3}-a_{1}}{a_{3}-a_{2}}\left(1-m \log \frac{\left(a_{2}-z\right)\left(a_{3}-a_{1}\right)}{\left(a_{1}-z\right)\left(a_{3}-z\right)}+O\left(m^{2}\right)\right) .
$$

It is tempting to interpret this effect as saying that the wiggles in the string get stretched when this pulls on the black hole, but we have not pursued this interpretation further.

While it does not seem feasible to identify in a unique manner the mass and acceleration of the black hole, we note that when the black hole-rod length $a_{2}-a_{1}$ is small

\footnotetext{
${ }^{5}$ We may equivalently say that we are matching the metrics induced on a surface at constant $z<a_{1}$.
} 
(much smaller than $a_{3}-a_{2}$ ) and $m$ is small, on general grounds we might expect to identify the ratio of surface gravities as

$$
\frac{\kappa_{R}}{\kappa_{\mathrm{BH}}} \simeq 4 G M A .
$$

In this limit $C$ is close to 1 so we can identify the string tension from (2.6). If $\varepsilon-T \ll T$ we find that Newton's second law

$$
T \approx M A
$$

is recovered. It is interesting to observe that for the $C$ metric $(m=0)$ the identity $1-C^{-1}=\kappa_{R} / \kappa_{\mathrm{BH}}$ is exactly satisfied.

\section{B. Pushing with struts}

Now we place a finite Levi-Civita rod along $a_{2}<z<a_{3}$ while leaving the semi-infinite axis $z<a_{1}$ exposed. If the latter is nonsingular, then, as we will see below, the segment $a_{2}<z<a_{3}$ must support a conical excess angle instead of a deficit angle, and hence we find a LeviCivita strut pushing on the black holes. This configuration has the advantage that, since the strut has finite length, we expect the metric to be asymptotically flat at spatial infinity. Furthermore, as discussed at the end of the previous section, the positive pressure along the strut need not imply a violation of energy conditions as long as $m$ is sufficiently large. This is unlike in the $C$ metric with a strut, which always violates positivity of energy and therefore makes the solutions manifestly unphysical.

The rod structure is as in Fig. 2. The metric functions are

$$
e^{2 U}=\mu_{1}\left(\frac{\mu_{3}}{\mu_{2}}\right)^{1-m},
$$

and

$$
\begin{aligned}
e^{2 \nu}= & \left(\frac{\mu_{3}}{\mu_{2}}\left(\frac{\mu_{1} \mu_{2}+\rho^{2}}{\mu_{1} \mu_{3}+\rho^{2}}\right)^{2}\left(\frac{\left(\mu_{2} \mu_{3}+\rho^{2}\right)^{2}}{\left(\mu_{2}^{2}+\rho^{2}\right)\left(\mu_{3}^{2}+\rho^{2}\right)}\right)^{1-m}\right)^{1-m} \\
& \times \frac{\mu_{1}}{\mu_{1}^{2}+\rho^{2}} .
\end{aligned}
$$

In contrast to the $C$ metric, when $m \neq 0$ the geometry is locally inequivalent to our previous solution where the strings run to infinity. Along $\rho=0$ we now have an exposed axis at $z<a_{1}$, a black hole horizon at $a_{1}<z<$ $a_{2}$, a Levi-Civita strut with rod density $m / 2 G$ at $a_{2}<z<$ $a_{3}$, and an acceleration horizon at $a_{3}<z$.

$$
\begin{array}{lll}
a_{1} & a_{2} & a_{3}
\end{array}
$$

FIG. 2. Weyl rod structure for the solution with a finite LeviCivita strut pushing the black hole. The rod at $a_{2}<z<a_{3}$ has linear density $m / 2 G$. The rods at $a_{1}<z<a_{2}$ and $z>a_{3}$ have linear density $1 / 2 G$.
The area and surface gravities of the horizons take the same form as in Eqs. (3.8), (3.9), and (3.11), but now regularity at the exposed axis $z<a_{1}$ requires

$$
C=1 \text {. }
$$

We can then expect an excess angle along the Levi-Civita rod. Indeed, for small $\rho$ and $a_{2}<z<a_{3}$ we find

$$
\begin{aligned}
e^{2 U} \simeq & \rho^{2 m} 2^{1-2 m} \frac{\left(\left(a_{3}-z\right)\left(z-a_{2}\right)\right)^{1-m}}{z-a_{1}} \\
e^{2 \nu} \simeq & \rho^{2 m(m-1)} \frac{2^{-1+2 m-2 m^{2}}}{\left(z-a_{1}\right)^{2 m-1}} \\
& \times\left(\frac{\left(a_{3}-a_{2}\right)^{2-2 m}\left(\left(a_{3}-z\right)\left(z-a_{2}\right)\right)^{-1+2 m}}{\left(a_{1}-a_{3}\right)^{2}}\right)^{1-m}
\end{aligned}
$$

and we can define a $z$-dependent $C$ parameter along this rod like we have done above. For small $m$ we find

$$
\begin{aligned}
\hat{C}(z)= & \frac{a_{3}-a_{2}}{a_{3}-a_{1}}\left(1+m \log \frac{\left(a_{3}-a_{1}\right)\left(a_{3}-z\right)\left(z-a_{2}\right)}{\left(z-a_{1}\right)\left(a_{3}-a_{2}\right)^{2}}\right. \\
& \left.+O\left(m^{2}\right)\right),
\end{aligned}
$$

which is smaller than 1 , reflecting the need of a negative tension (pressure) to push the black holes. The rest of the analysis can be carried out as in the previous solution and we omit it.

\section{Strings and struts}

Clearly, one can construct a larger class of metrics with a Levi-Civita rod at $z<a_{1}$ with density $m_{L} / 2 G$ and another rod at $a_{2}<z<a_{3}$ with density $m_{R} / 2 G$. The construction of these solutions is straightforward, and the metric functions are

$$
e^{2 U}=\rho^{2 m_{L}} \mu_{1}^{1-m_{L}}\left(\frac{\mu_{3}}{\mu_{2}}\right)^{1-m_{R}}
$$

and

$$
\begin{aligned}
e^{2 \nu}= & \left(\frac{\mu_{3}}{\mu_{2}}\right)^{1-m_{R}}\left(\frac{\left(\mu_{2} \mu_{3}+\rho^{2}\right)^{2}}{\left(\mu_{2}^{2}+\rho^{2}\right)\left(\mu_{3}^{2}+\rho^{2}\right)}\right)^{\left(1-m_{R}\right)^{2}} \\
& \times\left(\frac{\mu_{1} \rho^{-2 m_{L}}}{\left(\mu_{1}^{2}+\rho^{2}\right)^{1-m_{L}}}\left(\frac{\mu_{1} \mu_{2}+\rho^{2}}{\mu_{1} \mu_{3}+\rho^{2}}\right)^{2\left(1-m_{R}\right)}\right)^{1-m_{L}} .
\end{aligned}
$$

Since there is no exposed axis there does not seem to be any preferred value for the parameter $C$. This is then a fiveparameter family of solutions. Their analysis does not introduce any other important novelties so we shall not dwell on it. 


\section{OUTLOOK}

We have exhibited several new families of explicit solutions that describe black holes accelerating under the pull or push of a stringlike object. Their construction is fairly straightforward and our aim has been to underscore that these solutions can have physical significance, in particular, when strings pull on the black holes. One important feature is that, even if the Levi-Civita string (or strut) is strongly singular, it can end on the black hole without destroying the regularity of the horizon (away from the touch point). This feature was not a priori obvious, but it follows essentially from the properties of Weyl rod structures: close to a "horizon rod" the geometry is always of Rindler type (as we have explicitly exhibited). This is indeed the reason that, while we have not performed a detailed analysis of the extension of the solutions across the horizons, we do expect that this poses no difficulty. When the self-gravity of the string is weak (and hence the acceleration is small) it can be regarded as the zerothickness limit of a wiggly cosmic string, but it may also correspond to other nonsingular sources. The main difficulties in interpreting this solution and identifying its physical parameters stem from its unconventional asymptotics. But this is a problem only if we consider the string to be infinitely long, and if the solution is taken to approximate only a portion of a closed loop of string then the asymptotic behavior will be improved. On the other hand, the solutions with finite struts are presumably spatially asymptotically flat.

The existence and properties of these solutions raise a number of suggestions for future work:

String sources and cylindrical shells in the accelerating black hole solution.-We have not investigated the regularization of the Levi-Civita string in the accelerating black hole spacetime, but there are reasons to expect that this should not be problematic. In terms of wiggly strings, looking sufficiently close to the black hole one may resolve the wiggles and use the analysis of [19] to conclude that the vortex string can pierce the black hole. It would remain to solve the problem of how the wiggly structure extends to all the length of the string, possibly with $z$-dependent effective parameters as suggested by our analysis above. One may also replace the Levi-Civita string with a tubular shell. It does seem possible to cut the solution at some $\rho=$ $\rho(z)$ in a region $z \leq z_{s}$, with $z_{s}<a_{2}$, and replace the interior with a smooth spacetime, so the Levi-Civita string is replaced by an empty cylindrical shell that ends on the black hole. Israel's construction will yield the shell stress tensor. Stationarity demands that it be orthogonal to the null generator of the horizon $k$, i.e., $k^{\mu} k^{\nu} T_{\mu \nu}=0$. Other than this, in the absence of a specific model for the shell there do not seem to be any restrictions on its stress tensor.

Black hole charge and pair creation.-The impossibility of matching the black hole and acceleration temperatures prevents the construction of a Euclidean instanton that would mediate the snapping of the string by spontaneous formation of a pair of black holes at its end points. Extending our solution to include black hole charge should allow one to lower the black hole temperature to match the acceleration temperature, as in [3], and then study this process. For black holes in Kaluza-Klein theory the construction of this solution should be rather straightforward given the integrability of the five-dimensional equations.

AdS and black holes on branes.-There does not seem to exist any obstacle of principle to extending our solutions to include a (negative) cosmological constant, even if in practice finding exact solutions might not be feasible (for instance, inverse scattering techniques are unavailable for this case). At any rate, with these solutions one could investigate extensions of the construction of [4] of black holes localized on a Randall-Sundrum two-brane. Note, however, that the existence of solutions to Israel's junction conditions for a vacuum brane, i.e., one with extrinsic curvature proportional to its induced metric, is not guaranteed. Also, if the additional parameter in the solutions allowed one to construct a continuous family of black holes localized on a two-brane, this might seem to entail a continuous violation of uniqueness of black holes on the brane. However, although we are not aware of any theorems against this, it is unlikely to be realized in this manner since the Levi-Civita string ("hidden behind the brane") presumably makes it impossible to have flat asymptotics along the brane directions.

Global structure and gravitational radiation.-In this paper we have not attempted to study the maximal analytic extension and global structure of these solutions, but there may be more to this than a point of mathematical rigor. In particular it should be interesting to study the extension beyond the Rindler horizon of the solution with pulling strings to describe the "roof" in the Penrose diagram, where the radiative properties of the spacetime become apparent (see the second reference in [2]). The Levi-Civita string is absent from this region and so it may be interesting to study whether the asymptotic geometry at null infinity is better behaved. If radiation at infinity can be suitably characterized, this may provide an interesting extension of the class of boost-rotation symmetric radiative spacetimes. On the other hand, the solutions with struts probably have worse asymptotic behavior in the "roof."

Nonuniform rod density.-The only parameter that must be fixed in order to avoid singularities on the exposed axis is $C$, which amounts to a simple rescaling of $\phi$, and which in the linearized limit corresponds to the string tension. Thus it would seem possible to construct Weyl solutions analogous to the ones we have studied, with naked singularities only at the pulling string, where the rod at $z<a_{1}$ would have $z$-dependent density $m(z)$ [varying in the range $(0,1 / 2)$ or possibly $(0,1)]$, while $C$ remains constant. Obviously the same could be done with finite struts. In general, explicit solutions could be found presumably only 
up to quadratures, but their properties might perhaps still be analyzable. This would give a one-function family of accelerating black holes. It is conceivable that if $m$ approaches zero sufficiently fast as $z \rightarrow-\infty$ the asymptotic behavior might be as in the $C$ metric.

Accelerating black holes in higher dimensions.-No exact solution for accelerating black holes in $D>4$ is known. Reference [20] solved the perturbation equations for a $D>$ 4 Schwarzschild black hole to give it uniform acceleration and found a solution with a distributional linear source accelerating the black hole.

Let us reexamine this problem in light of what we have learned in four dimensions. In the class of metrics we have analyzed, the $C$ metric is singled out as the one where the string has a milder singularity at the axis and also has better-behaved (locally flat) asymptotics. In contrast, in $D \geq 5$ we would expect the asymptotic behavior to be good for all string sources with finite energy density and tension: the gravitational field in directions transverse to the string falls off like $\sim 1 / r^{D-4}$. Near the string, however, there are significant differences between sources. Requiring the symmetry $\mathbb{R}_{t} \times \mathbb{R}_{z} \times S O(D-2)$, the static, cylindrically symmetric stringlike solutions to the vacuum Einstein equations can be obtained as a particular case of solutions in [21] (or in $D=5$ by uplifting the solutions in [22]) to find

$$
\begin{aligned}
d s^{2}= & -f^{[(D-3) \varepsilon-T] / \mu} d t^{2}+f^{[(D-3) T-\varepsilon] / \mu} d z^{2} \\
& +f^{-(D-5) /(D-4)-(\varepsilon+T) / \mu} d r^{2} \\
& +f^{1 /(D-4)-(\varepsilon+T) / \mu} r^{2} d \Omega_{(D-3)}
\end{aligned}
$$

with

$$
\begin{aligned}
& f=1-\frac{16 \pi G}{(D-4)(D-2) \Omega_{(D-3)}} \frac{\mu}{r^{D-4}}, \\
& \mu=\sqrt{(D-4)(D-2)\left(\varepsilon^{2}+T^{2}-\frac{2}{D-3} \varepsilon T\right)} .
\end{aligned}
$$

We may regard these as the $D$-dimensional versions of the Levi-Civita strings. The two parameters $\varepsilon, T$ are the energy density and tension measured at asymptotic infinity. They coincide with the energy density and tension of the sources for the linearized approximation to the solutions. When $T=\varepsilon /(D-3)$ we recover the black string, ${ }^{6}$ but in all other cases the solutions present naked singularities where $f=0$, including, in particular, the strings with Lorentzinvariant world sheet, $\varepsilon=T$ [23]. ${ }^{7}$ One might nevertheless expect that, like in four dimensions, all of these strings

\footnotetext{
${ }^{6}$ Observe that this contains the conical-defect strings for $D=$ 4.

${ }^{7}$ The case $\varepsilon=T /(D-3)$ has conical singularities when $-\infty<z<\infty$. Note also that only when $\varepsilon>(D-3) T$ or $\varepsilon<$ $T /(D-3)$ does the angular $S^{D-3}$ shrink to zero at the singularity.
}

with $T>0$ should be able to accelerate a massive object. The black string might be more appealing physically, but it is not known whether it can pierce a black hole horizon in a nonsingular manner. ${ }^{8}$

This suggests that in $D>4$, as in four dimensions, a family of accelerating black hole solutions should exist with at least three independent parameters, for the black hole mass and the string energy density and tension, with an open set of their values being potentially useful for physical applications. But, unlike in four dimensions, the asymptotic behavior does not seem to single out any specific solution, so in this respect they all appear to be on a similar footing and different pulling strings may be relevant to different problems. It is even possible that strings with nonuniform density need to be considered, e.g., in order to satisfy the junction conditions on the brane as suggested by the results of [20]. One may also consider struts pushing on the black holes, but they are always nakedly singular since there are no "black struts."

\section{ACKNOWLEDGMENTS}

This work was supported by DURSI 2009 SGR 168, MEC FPA 2007-66665-C02, and CPAN CSD2007-00042 Consolider-Ingenio 2010. J. C. was also supported in part by FPU Grant No. AP2005-3120.

\section{APPENDIX: THE SOLUTIONS IN $(x, y)$ COORDINATES}

The $C$ metric is customarily written not in Weyl coordinates but in a set of coordinates $(x, y)$ adapted to uniformly accelerated motion. In order to write our solutions in these coordinates, we perform the change

$$
\begin{aligned}
\rho & =\frac{2}{A^{\alpha}(x-y)^{2}} \sqrt{\left(1-x^{2}\right)\left(y^{2}-1\right)(1+\nu x)(1+\nu y),} \\
z & =\frac{(1-x y)(2+\nu(x+y))}{A^{\alpha}(x-y)^{2}},
\end{aligned}
$$

and

$$
a_{1}=-\frac{\nu}{A^{\alpha}}, \quad a_{2}=\frac{\nu}{A^{\alpha}}, \quad a_{3}=\frac{1}{A^{\alpha}},
$$

which can be generically applied to any Weyl solution with two finite rods. The parameter $A$ fixes the overall scale, and its exponent is

$$
\begin{aligned}
& \alpha=\frac{2}{1+m} \text { for solutions with strings, } \\
& \alpha=2 \text { for solutions with struts. }
\end{aligned}
$$

We take $y \leq-1,-1 \leq x \leq 1$, and $0<\nu<1$.

${ }^{8}$ The methods of [24] should be of help here. 
The functions $\mu_{i}$ in (3.4) then become

$$
\begin{aligned}
& \mu_{1}=2 \frac{(x-1)(1+y)(1+\nu y)}{A^{\alpha}(x-y)^{2}}, \\
& \mu_{2}=2 \frac{(x-1)(1+y)(1+\nu x)}{A^{\alpha}(x-y)^{2}}, \\
& \mu_{3}=2 \frac{\left(y^{2}-1\right)(1+\nu x)}{A^{\alpha}(x-y)^{2}} .
\end{aligned}
$$

Defining $G(\xi)=\left(1-\xi^{2}\right)(1+\nu \xi)$, the metric with strings of Sec. III A reads

$$
\begin{aligned}
d s^{2}= & \frac{2}{A^{2}(x-y)^{2}}\left[G(y)\left(\frac{2 G(x)}{(x-y)^{2}} \frac{1-y}{1-x}\right)^{m} d t^{2}\right. \\
& \left.+G(x)\left(\frac{2 G(x)}{(x-y)^{2}} \frac{1-y}{1-x}\right)^{-m} \frac{d \phi^{2}}{\bar{C}^{2}}\right] \\
& +\frac{Y}{A^{2}}\left(-\frac{d y^{2}}{G(y)}+\frac{d x^{2}}{G(x)}\right),
\end{aligned}
$$

where

$$
\begin{aligned}
Y= & 2\left(\frac{1-\nu}{x-y}\right)^{2} \\
& \times\left[\frac{(1-x)(1-y)(2+\nu(1+x+y-x y))^{2-m}}{2 G(x)}\right. \\
& \left.\times\left(\frac{G(x)(1-y)}{(1-x)(x-y)}\right)^{m}\right]^{m},
\end{aligned}
$$

and

$$
\bar{C}=\frac{1}{2^{m}} \frac{(1+\nu)^{1-m}}{1-\nu} .
$$

For the solution with struts of Sec. III B we get

$$
\begin{aligned}
d s^{2}= & \frac{2}{A^{2}(x-y)^{2}}\left[G(y)\left(\frac{1-x}{1-y}\right)^{m} d t^{2}+G(x)\right. \\
& \left.\times\left(\frac{1-x}{1-y}\right)^{-m} d \phi^{2}\right]+\frac{\Upsilon}{A^{2}}\left(-\frac{d y^{2}}{G(y)}+\frac{d x^{2}}{G(x)}\right),
\end{aligned}
$$

now with

$$
Y=2\left(\frac{1-\nu}{x-y}\right)^{2}\left(\frac{[-(x+y+\nu(1+x y))(2+\nu(-1+x+y+x y))]^{2-m}}{4(1-\nu)^{2(2-m)}((1-x)(1-y))^{1-m}}\right)^{m}
$$

When $m=0$ both solutions reduce, up to a constant rescaling of coordinates, to the uncharged $C$ metric with the factorized form for $G(\xi)$ first given in [25] (see also [26]).

[1] T. Levi-Civita, Rend. Acc. Lincei 27, 343 (1918); H. Weyl, Ann. Phys. (Leipzig) 364, 185 (1919); W. Kinnersley and M. Walker, Phys. Rev. D 2, 1359 (1970).

[2] For instance, see J. Bičák, Proc. R. Soc. A 302, 201 (1968); J. Bičák and B. G. Schmidt, Phys. Rev. D 40, 1827 (1989); J. Bičák, P. Reilly, and J. Winicour, Gen. Relativ. Gravit. 20, 171 (1988); J. Bičák and A. Pravdová, J. Math. Phys. (N.Y.) 39, 6011 (1998); J. Bičák and V. Pravda, Phys. Rev. D 60, 044004 (1999).

[3] Some relevant references include D. Garfinkle and A. Strominger, Phys. Lett. B 256, 146 (1991); D. Garfinkle, S. B. Giddings, and A. Strominger, Phys. Rev. D 49, 958 (1994); F. Dowker, J. P. Gauntlett, D. A. Kastor, and J. H. Traschen, Phys. Rev. D 49, 2909 (1994); S. W. Hawking, G. T. Horowitz, and S.F. Ross, Phys. Rev. D 51, 4302 (1995); S. W. Hawking and S. F. Ross, Phys. Rev. Lett. 75, 3382 (1995); R. Emparan, Phys. Rev. Lett. 75, 3386 (1995); D. M. Eardley, G. T. Horowitz, D. A. Kastor, and J. H. Traschen, Phys. Rev. Lett. 75, 3390 (1995); R. Gregory and M. Hindmarsh, Phys. Rev. D 52, 5598 (1995).

[4] R. Emparan, G. T. Horowitz, and R. C. Myers, J. High Energy Phys. 01 (2000) 007; 01 (2000) 021; R. Emparan, R. Gregory, and C. Santos, Phys. Rev. D 63, 104022 (2001); M. Anber and L. Sorbo, J. High Energy Phys.
07 (2008) 098; R. Emparan and G. Milanesi, J. High Energy Phys. 08 (2009) 012.

[5] R. Emparan and H. S. Reall, Phys. Rev. Lett. 88, 101101 (2002); R. Emparan, J. High Energy Phys. 03 (2004) 064; E. Teo, Phys. Rev. D 73, 024016 (2006).

[6] T. Levi-Civita, Rend. Acc. Lincei 28, 101 (1919).

[7] V. Belinski and E. Verdaguer, Gravitational Solitons (Cambridge University Press, Cambridge, England, 2001).

[8] W. B. Bonnor, in On Einstein's Path: Essays in Honor of Engelbert Schucking, edited by A. Harvey (Springer, New York, 1999), p. 113.

[9] J. Stachel, J. Math. Phys. (N.Y.) 25, 338 (1984).

[10] T. G. Philbin, Classical Quantum Gravity 13, 1217 (1996).

[11] A.Z. Wang, M. F. A. da Silva, and N. O. Santos, Classical Quantum Gravity 14, 2417 (1997).

[12] L. Herrera, N. O. Santos, A. F. F. Teixeira, and A. Z. Wang, Classical Quantum Gravity 18, 3847 (2001).

[13] J. Bičák and M. Žofka, Classical Quantum Gravity 19, 3653 (2002)

[14] J. Bičák, T. Ledvinka, B. G. Schmidt, and M. Žofka, Classical Quantum Gravity 21, 1583 (2004).

[15] W. Israel, Nuovo Cimento B 44S10, 1 (1966); 48, 463 (1967).

[16] A. Vilenkin and E.P.S. Shellard, Cosmic Strings and Other Topological Defects (Cambridge University Press, 
Cambridge, England, 1994).

[17] R. Emparan and H.S. Reall, Phys. Rev. D 65, 084025 (2002).

[18] K. Dutta, S. Ray, and J. Traschen, Classical Quantum Gravity 23, 335 (2006).

[19] A. Achúcarro, R. Gregory, and K. Kuijken, Phys. Rev. D 52, 5729 (1995).

[20] H. Kodama, Prog. Theor. Phys. 120, 371 (2008).

[21] G. W. Gibbons and K.i. Maeda, Nucl. Phys. B298, 741
(1988).

[22] A. G. Agnese and M. La Camera, Phys. Rev. D 31, 1280 (1985).

[23] R. Gregory, Nucl. Phys. B467, 159 (1996).

[24] R. Emparan, T. Harmark, V. Niarchos, and N. A. Obers, J. High Energy Phys. 03 (2010) 063.

[25] R. Emparan, Nucl. Phys. B490, 365 (1997).

[26] K. Hong and E. Teo, Classical Quantum Gravity 20, 3269 (2003). 